\title{
Mejora del rendimiento académico del alumnado de Farmacología mediante la utilización de metodologías activas y recursos on-line.
}

\author{
M. Asier Garro ${ }^{a}$, Jaione Lacalle ${ }^{b}, M^{a}$ Teresa Barandiaran ${ }^{c}, M^{a}$ Jose Uranga $^{d}, M^{a}$ \\ Nieves $\operatorname{Aja}^{\mathrm{e}}$ y Jesús Rubio-Pilarte ${ }^{\mathrm{f}}$ \\ a, b, c, d, e y f Departamento de Enfermería II. Facultad de Medicina y Enfermería. Sección Donostia. \\ a(mikelasier.garro@ehu.eus), b(jaione.lacalle@ehu.eus), (mariateresa.barandiaran@ehu.eus), \\ d(mariajose.uranga@ehu.eus) ${ }^{\text {en }}$ (nieves.aja@ehu.eus) y f(jesus.rubio@ehu.eus).
}

\begin{abstract}
The subject of Pharmacology in the Nursing degree meets the learning needs for the future professional activity of Nurses, as important as the correct administration of drugs or the prevention of adverse events through knowledge of the mechanisms of action and adverse effects of drugs. . The objective of this study is to analyze the possible benefit that the implementation of on-line resources and active methodologies such as Socrative ${ }^{\circledR}$ questionnaires or the flipped classroom have on the acquisition of these competences by the second-year students of the Nursing degree. Three cohorts have been analyzed with a progressive implementation of these resources and teaching methodologies. The results show a clear improvement in the academic performance of the students in all the analyzed competences with an acceptable cost-benefit.
\end{abstract}

Keywords:

pharmacology, drug dosage calculation, academic performance, on-line resources, flipped classroom

\footnotetext{
Resumen

La asignatura de Farmacología en el grado de Enfermería cubre necesidades de aprendizaje para la futura actividad profesional del personal de Enfermería tan importantes como la correcta administración de fármacos o la prevención de eventos adversos mediante el conocimiento de los mecanismos de acción y efectos adversos de los fármacos. El objetivo del presente trabajo es analizar el posible beneficio que la implantación de metodologías activas y recursos on-line así como cuestionarios Socrative ${ }^{\circledR} y$ el aula invertida tienen sobre la adquisición de dichas competencias por parte del alumnado de segundo curso del grado de Enfermería. Se han analizado tres cohortes con una implantación progresiva de dichas metodologías y recursos docentes. Los resultados demuestran una clara mejoría en el rendimiento académico del alumnado en todas las competencias analizadas con un coste-beneficio en cuanto a esfuerzo del alumnado y mejora en el rendimiento académico asumible.
} 
Mejora del rendimiento académico del alumnado de Farmacología mediante la utilización de metodologías activas y recursos on-line.

Palabras clave: farmacología, habilidad en cálculo de dosis, rendimiento académico, materiales on-line, aula invertida

\section{Introducción}

El Grado de Enfermería en la Sección Donostia de la Facultad de Medicina y Enfermería de la UPV/EHU se implantó en el curso académico 2010-2011. Ya desde el inicio, ha existido un gran interés por parte del profesorado y de la Dirección del Centro para coordinar las diferentes asignaturas tanto horizontal como verticalmente con el objetivo de optimizar el esfuerzo requerido al alumnado en la adquisición de las competencias del Grado de Enfermería. De este modo, se procedió a medir, en alumnado voluntario, la carga de trabajo que suponían las diferentes actividades "no presenciales" a realizar (fuera del horario establecido) en el marco del creditaje de las diferentes asignaturas. Todo ello conllevó a establecer reuniones de coordinación con el objetivo de no sobrecargar al alumnado en determinadas fechas y poder así hacer frente con garantías a la adquisición de las competencias de las diferentes asignaturas. La asignatura Farmacología en el Grado de Enfermería cubre aspectos tan importantes para la profesión de Enfermería como son las competencias relacionadas con la correcta administración de fármacos: el conocimiento de los mecanismos de acción, las dosis terapéuticas, los efectos terapéuticos y los efectos adversos de los fármacos. En la Universidad del País Vasco/Euskal Herriko Unibertsitatea (UPV/EHU) esta asignatura se imparte en el segundo curso tras la impartición en primer curso de diferentes asignaturas necesarias para integrar los conocimientos que se imparten en Farmacología, como por ejemplo: Estructura y Función del Cuerpo Humano I y III (Anatomía Humana y Fisiología Humana) así como, Sistemas de la Información y Análisis de Datos, asignatura mediante la cual el alumnado conoce el funcionamiento de diferentes programas informáticos y Bases de Datos. Dentro de la asignatura de Farmacología, el cálculo correcto de las dosis es una competencia clave que es imprescindible superar para aprobar la asignatura. El análisis de los resultados en esta competencia durante el periodo comprendido entre 2012-2016 reveló un descenso importante en el número de alumnas/os que superaban dicha competencia en primera convocatoria (Garro y cols, 2018). De igual modo, la literatura corrobora la existencia de un problema asociado a la correcta administración de dosis, siendo un 15\% de errores detectados por Cousins y cols. (2012), incrementándose a un 25\% en el caso de pacientes pediátricos (Manias y cols, 2013). Este hecho nos hizo reflexionar y tras revisar la literatura, planteamos como elemento de mejora para el aprendizaje del alumnado la elaboración de material on-line. Dicho material fue utilizado por el alumnado en el curso académico 2017-2018 en las horas "no presenciales" sin alterar la metodología docente que seguía siendo de tipo deductivo. Los resultados obtenidos mostraron una mejora del aprendizaje en torno al cálculo de dosis (manuscrito en preparación) pero disminuyeron los relacionados con otras competencias como, por ejemplo, el conocimiento de los efectos adversos más importantes (Lacalle y cols, aceptado en CINDU, Vigo 2019). En este mismo sentido, Gill y cols (2019). encontraron que la implantación de recursos on-line mejoraron los resultados académicos del alumnado en la 
asignatura de Farmacología. Todo ello, nos ha obligado a un replanteamiento del modelo de aprendizaje utilizado.

\section{Objetivos}

Los objetivos del presente estudio son:

Comprobar si metodologías docentes predominantemente inductivas (aula invertida) y activas (cuestionarios Socrative) mejoran los resultados en las pruebas finales.

Estudiar el coste-beneficio para el alumnado de dichas metologías en cuanto a rendimiento académico se refiere.

\section{Desarrollo de la innovación}

Una vez analizadas las diferentes metodologías docentes existentes, durante el curso académico 2018-2019 se procedió a implantar diversas metodologías activas: 5 pruebas evaluativas Socrative ${ }^{\circledR}$ a realizar en horario presencial que complementaban las 110 actividades on-line "Hot-pot" que se habían desarrollado para el curso académico 20172018, 22 actividades Hot-pot de 15 preguntas de elección múltiple cada una, 66 actividades correspondientes a frases del temario con huecos que el alumnado debía rellenar y finalmente, en las 22 actividades de emparejamiento de diferentes enunciados. En todas estas actividades que se realizaban en horas no presenciales, el alumnado recibía el feedback inmediatamente. Las pruebas evaluativas mediante cuestionarios de 20 preguntas cada una eran acumulativas, es decir, el temario cada vez era más extenso a medida que se avanzaba en la realización de dichos cuestionarios.

Por otro lado, en el curso académico 2018-2019 a diferencia del anterior, se ha procedido a cambiar la metodología docente en lo relativo a la competencia del correcto cálculo de dosis. De este modo, en este pasado curso académico se ha procedido a impartir en un primer seminario la teoría mínima necesaria en cuanto a unidades de medida y conversión entre las mismas. A continuación el alumnado semanalmente ha realizado a lo largo de 14 semanas en horas no presenciales ocho Hot-pots de 10 ejercicios de dosificación cada uno y cuatro paquetes multimedia con 15 ejercicios de dosificación cada uno (los mismos ejercicios que en el curso académico 2017-2018) pero con una metodología activa como es el aula invertida. Una vez realizados los ejercicios en horas presenciales se procedía al debate y resolución de los ejercicios que hubieran generado problemas al alumnado. Para ello se realizaba una puesta en común de los diferentes abordajes empleados para la realización de los ejercicios y quedaban, por tanto, a disposición del alumnado diferentes abordajes conceptuales de la resolución de los mismos. Los ejercicios a medida que se realizaban semanalmente quedaban a disposición del alumnado para que los volvieran a realizar cuando mejor lo consideraran. Los resultados del curso 2016-2017, previos a la introducción de estas metodologías, se utilizarán como control.

Se solicitó la participación voluntaria y el consentimiento informado a los/las estudiantes pertenecientes a las tres cohortes estudiadas (2016-2017, 2017-2018 y 2018-2019). Se les notificó que la participación era voluntaria y que podían retirar su consentimiento en 
cualquier momento. Este estudio ha sido aprobado por el Comité de Ética de la UPV/EHU (código: M10_2018_131). En el estudio participaron un total de 273 estudiantes $(68,76 \%$ de estudiantes de enfermería elegibles) que se distribuyeron de la siguiente manera: 66 estudiantes de 135 del año académico 2016-2017, 105 estudiantes de un total de 135 estudiantes de 2017-2018 año académico y 102 estudiantes de un total de 127 estudiantes en el año académico 2018-2019. Los porcentajes de mujeres fueron $86.36 \%$, 87.62\% y $86.27 \%$ respectivamente. La edad promedio de los participantes fue de 20.4 años (mediana: 19.56, D.E: 3.11; rango: 18-42 años). Para el estudio del coste-efectividad de estas metodologías se analizará la relación entre el tiempo empleado para el estudio, medido en horas, y la calificación obtenida en los ejercicios realizados.

Las variables dependientes analizadas han sido: horas empleadas en la realización de los Hot-Pot correspondientes a la teoría, horas empleadas en el repaso de los ejercicios de cálculo de dosificación, nota teórica obtenida en el examen final, nota obtenida en el apartado de cálculo de dosis del examen final, número de aprobados en la parte teórica del examen final así como el número de alumnos/as con un 100\% de aciertos en la parte correspondiente al cálculo de dosis del examen final (datos estos últimos utilizados para el cálculo de los odds ratio). Por otro lado, las variables independientes utilizadas han sido: curso académico y línea idiomática. Los resultados obtenidos en el examen teórico del curso académico 2018-2019 correspondientes a la línea idiomática de euskera han sido excluidos del estudio debido a que se ha comprobado mediante la inserción de preguntas control (nuevas preguntas acerca de efectos adversos a las ya incluidas anteriormente) que el alumnado disponía de las preguntas del examen del curso anterior 2017-2018 previo al examen final.

El análisis de los resultados ha revelado que no se ajustan a una distribución normal por lo que se ha procedido a un análisis no paramétrico de los mismos. Se presentan los datos descriptivos (mediana, desviación estándar, rango y $\mathrm{N}$ válido) así como las correlaciones de Rho de Spearman, U de Mann-Whitney y los odds ratio entre las diferentes cohortes analizadas.

El paquete estadístico utilizado ha sido SPSS ${ }^{\circledR}$ para Windows 25.0 (SPSS INc., Chicago IL, EE.UU). Los valores de P inferiores a 0,05 se consideraron significativos. Por otro lado, en el caso de los odds ratio se han utilizado los intervalos de confianza para determinar la significancia de los mismos.

El ajuste no lineal se realizó mediante el programa Graph-Pad Prism ${ }^{\circledR}$ 5. Los datos se analizaron mediante regresión no lineal comparándose el ajuste a una recta y a una curva exponencial en los dos conjuntos de datos analizados.

\section{Resultados}

Los datos descriptivos de las variables dependientes por cursos académicos figuran en la tablas 1 y 2 . 
Tabla 1. Datos descriptivos de los resultados obtenidos en las variables analizadas.

\begin{tabular}{|c|c|c|c|c|c|c|c|c|c|c|}
\hline & & \multicolumn{3}{|c|}{$\begin{array}{l}\text { Horas empleadas } \\
\text { estudio teórico }\end{array}$} & \multicolumn{3}{|c|}{$\begin{array}{l}\text { Horas empleadas en } \\
\text { el repaso de los } \\
\text { ejercicios de } \\
\text { dosificación }\end{array}$} & \multicolumn{3}{|c|}{$\begin{array}{l}\text { Nota obtenida en } \\
\text { la parte teórica del } \\
\text { examen final }\end{array}$} \\
\hline \multirow{2}{*}{$\begin{array}{l}\text { Curso } \\
\text { académico }\end{array}$} & \multirow{2}{*}{$\begin{array}{c}\text { Línea } \\
\text { idiomática }\end{array}$} & & & & & & & & & \\
\hline & & Mediana & $\mathrm{DE}$ & $\mathrm{N}$ & Mediana & $\mathrm{DE}$ & $\mathrm{N}$ & Mediana & $\mathrm{DE}$ & $\mathrm{N}$ \\
\hline \multirow{2}{*}{$\begin{array}{l}2016- \\
2017\end{array}$} & Euskera & & & & & & & 5,64 & 2,81 & 44 \\
\hline & Castellano & & & & & & & 6,44 & 3,49 & 22 \\
\hline \multirow{2}{*}{$\begin{array}{l}2017- \\
2018\end{array}$} & Euskera & 2,40 & 2,59 & 66 & 2,50 & 3,49 & 66 & 6,51 & 2,16 & 53 \\
\hline & Castellano & 0,20 & 0,43 & 39 & 2,92 & 5,44 & 39 & 4,38 & 2,46 & 38 \\
\hline \multirow{2}{*}{$\begin{array}{l}2018- \\
2019\end{array}$} & Euskera & 2,87 & 2,95 & 69 & 2,08 & 2,70 & 70 & & & \\
\hline & Castellano & 3,67 & 2,74 & 31 & 0,83 & 4,89 & 32 & 6,77 & 2,09 & 32 \\
\hline
\end{tabular}

Tabla 2. Datos descriptivos de los resultados obtenidos en las variables analizadas.

\begin{tabular}{|c|c|c|c|c|c|c|c|c|}
\hline & & \multicolumn{3}{|c|}{$\begin{array}{l}\text { Nota obtenida en la } \\
\text { parte } \\
\text { correspondiente al } \\
\text { cálculo de dosis del } \\
\text { examen final }\end{array}$} & \multicolumn{2}{|c|}{$\begin{array}{c}\text { Número de } \\
\text { aprobados y } \\
\text { suspensos en la } \\
\text { parte teórica del } \\
\text { examen final }\end{array}$} & \multicolumn{2}{|c|}{$\begin{array}{l}\text { Número de } \\
\text { aciertos al } 100 \% \\
\text { en la parte } \\
\text { correspondiente al } \\
\text { cálculo de dosis } \\
\text { del examen final }\end{array}$} \\
\hline \multirow{2}{*}{$\begin{array}{l}\text { Curso } \\
\text { académico }\end{array}$} & \multirow{2}{*}{$\begin{array}{c}\text { Línea } \\
\text { idiomática }\end{array}$} & & & & & & & \\
\hline & & $\begin{array}{c}\text { Median } \\
\mathrm{a}\end{array}$ & $\mathrm{DE}$ & $\mathrm{N}$ & Aprobados & $\begin{array}{c}\text { Suspenso } \\
\mathrm{s}\end{array}$ & Aprobados & $\begin{array}{c}\text { Suspens } \\
\text { os }\end{array}$ \\
\hline \multirow{2}{*}{$\begin{array}{l}2016- \\
2017\end{array}$} & Euskera & 87,50 & 26,62 & 41 & 28 & 16 & 15 & 26 \\
\hline & Castellano & 87,50 & 29,69 & 21 & 14 & 8 & 4 & 17 \\
\hline \multirow{2}{*}{$\begin{array}{l}2017- \\
2018\end{array}$} & Euskera & 60,00 & 27,72 & 63 & 31 & 35 & 28 & 35 \\
\hline & Castellano & 60,00 & 26,52 & 39 & 17 & 22 & 18 & 21 \\
\hline \multirow{2}{*}{$\begin{array}{l}2018- \\
2019\end{array}$} & Euskera & 72,50 & 27,57 & 68 & & & 28 & 42 \\
\hline & Castellano & 100 & 34,58 & 32 & 24 & 8 & 20 & 12 \\
\hline
\end{tabular}

El análisis de los resultados del curso académico 2017-2018 donde se implantaron los recursos on-line reveló que las medianas de las notas obtenidas en la parte teórica y de 
cálculo de dosis no fueron significativamente diferentes con respecto al curso académico 2016-2017 (U de Mann-Whitney; N.S.). El Odds Ratio calculado sobre los aprobados de la parte teórica del examen final (evento positivo) reveló un descenso en el número de aprobados en la parte teórica del examen final con respecto al curso académico 2016-17 $(\mathrm{OR}=0,48$; IC: $0,26-0,90, \mathrm{P}<0.05)$. Sin embargo, hubo un aumento que no llego a ser estadísticamente significativo en el número de alumnos/as con un $100 \%$ de aciertos en la parte de cálculo de dosis del examen final ( $\mathrm{OR}=1,86$; IC: $0,96-3,62$; N.S.).

No obstante, al comparar los resultados obtenidos en el curso académico 2018-2019, donde se implantaron los cuestionarios Socrative ${ }^{\circledR}$, con respecto al curso académico 2017-2018 las horas empleadas para el repaso de la parte teórica aumentó significativamente $(\mathrm{P}<0.001)$. Sin embargo, aún siendo superior la mediana obtenida en la parte teórica del examen final del curso académico 2018-2019, esta diferencia no resultó ser estadísticamente significativa con respecto a los cursos académicos anteriores. A pesar de ello, el Odd Ratio correspondiente a los aprobados en la parte teórica del curso académico 2018-2019 reveló un aumento que únicamente resultó ser estadísticamente significativo con respecto al curso académico 2017-2018 ( $\mathrm{OR}=3,56$, IC: $1,47-8,65 ; \mathrm{P}<0.05)$. En cuanto a la parte de cálculo de dosis del examen final el Odd Ratio fue estadísticamente significativo $(\mathrm{OR}=2,01, \mathrm{IC}: 1,03-3,91)$ únicamente con respecto al curso académico 2016-2017.

Las horas empleadas en el repaso de los ejercicios on-line (parte teórica y cálculo de dosis) correlacionaron significativamente en el curso académico 2017-2018 con las notas obtenidas en las dos partes del examen analizadas $(\rho=0,351 ; \mathrm{P}<0.001 ; \mathrm{N}=91$ y $\rho=0,337$; $\mathrm{P}<0.001 ; \mathrm{N}=102$ para la parte teórica y cálculo de dosis respectivamente). En el curso académico 2018-2019, sin embargo, dichas correlaciones no resultaron estadísticamente significativas $(\rho=0,329 ; \mathrm{P}=0.071 ; \mathrm{N}=31$ y $\rho=0,187 ; \mathrm{P}=0.062 ; \mathrm{N}=100$ para la parte teórica y cálculo de dosis respectivamente).

El análisis de las horas totales en los diferentes cursos académicos (2017-2018 y 20182019) reveló una media de 5,58 horas empleadas para las dos partes del examen en el curso académico 2017-2018 y de 6,53 horas en el curso académico 2018-2019. La diferencia rozó la significancia estadística $(\mathrm{P}=0,051)$. Por otro lado, el análisis de las dos líneas idiomáticas reveló que si bien hubo diferencias entre los grupos de euskera y castellano en el curso académico 2017-2018, esta diferencia no resultó estadísticamente significativa. En el curso académico 2018-2019 no hubo diferencias entre las dos líneas idiomáticas.

Como se puede observar en la figura 1 la relación entre las horas empleadas en el repaso de la parte teórica y la nota obtenida en la parte teórica del examen final se ajustaron a una recta en el curso académico 2017-2018, mientras que los datos del curso académico 20182019 se ajustaron mejor a una curva exponencial. 
Figura 1. Horas empleadas en el repaso de los ejercicios de la parte teórica en los cursos académicos 2017-18 y 2018-2019
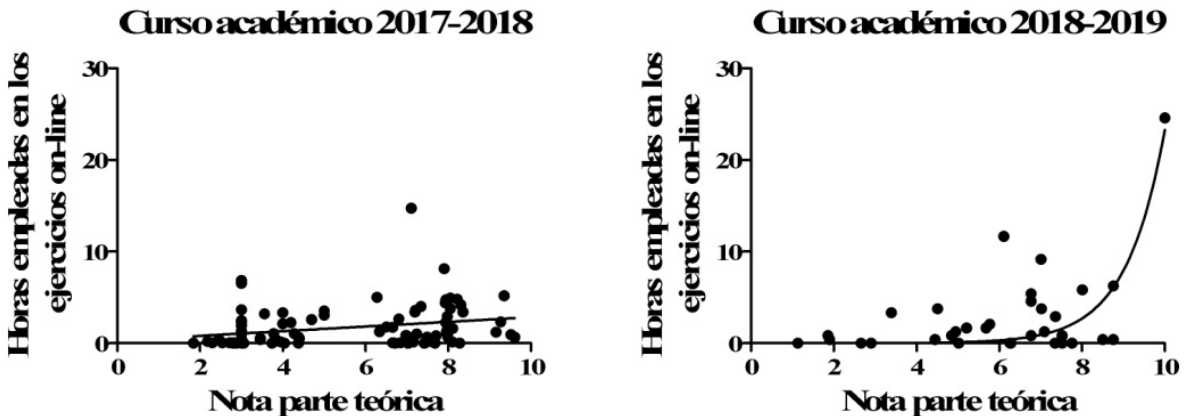

En lo referente a las correlaciones entre las horas empleadas en el repaso de los ejercicios de cálculo de dosis en los dos cursos académicos (2017-2018 y 2018-2019) los datos se ajustaron mejor a un modelo de curva exponencial (Figura 2).

Figura 2. Horas empleadas en el repaso de los ejercicios de cálculo de dosis en los cursos académicos 2017-18 y 2018-2019
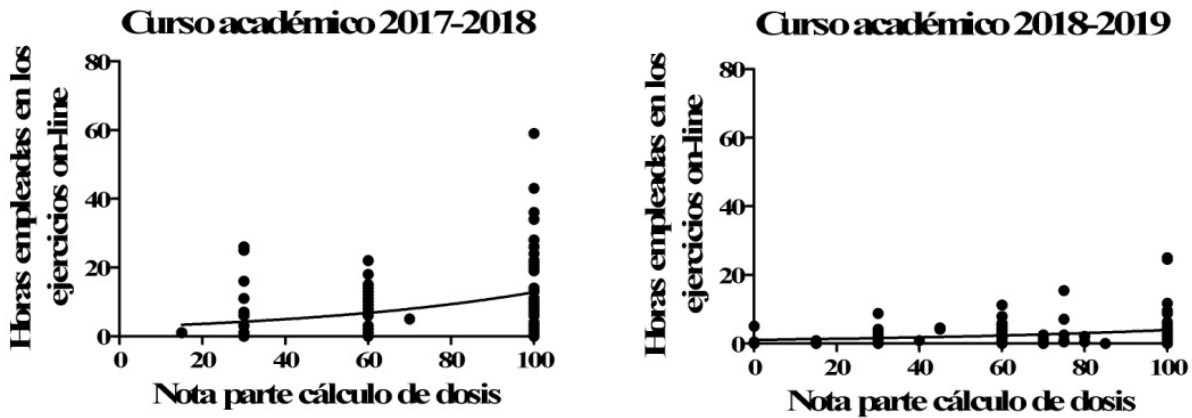

\section{Discusión y conclusiones}

Los resultados obtenidos en su conjunto revelan que mientras la implantación de recursos on-line en el curso académico 2017-2018 mejoró de una forma no significativa el rendimiento académico en la competencia de cálculo de dosis, conllevó una reducción en el rendimiento del alumnado en lo referente a la parte teórica del examen final. Hemos de tener en cuenta que durante el curso académico 2017-2018 la metodología docente empleada fue de tipo deductivo, basándose en la repetición de los ejercicios Hot-Pot planteados. Este hecho se puede apreciar claramente en la figura 2 donde el número de horas empleadas en los ejercicios de cálculo de dosis en el curso académico 2017-18 fue relativamente superior al del curso académico 2018-2019.

Sin embargo, la implantación de los cuestionarios acumulativos en materia a estudio (metodología activa) y el cambio de una metodología docente eminentemente deductiva a 
una inductiva conllevó una mejora en los resultados en cuanto al número de aprobados en la parte teórica así como en el número de alumnos/as con un 100\% de aciertos en el cálculo de dosis con respecto a los cursos académicos 2017-2018 y 2016-2017 respectivamente.

El análisis de la horas empleadas en la realización de los diferentes ejercicios tanto de la parte teórica como de la parte de cálculo de dosis, indica que nos movemos (5,58 y 6,53 horas en los dos cursos analizados) dentro de los parámetros establecidos en el plan de estudios, donde se prevén 90 horas de estudio no presenciales en la asignatura de Farmacología. Sin embargo, se ha de aclarar que a las horas empleadas en la realización de los ejercicios hay que sumarles las horas previas de preparación para la correcta realización de los mismos. El coste beneficio de la implantación combinada de metodologías activas y recursos on-line con respecto a los recursos on-line revelan que si bien existe un aumento no significativo en el número de horas empleadas en el repaso de los ejercicios, este aumento se debió a un mayor estudio de la parte teórica promovida por la realización de los cuestionarios Socrative ${ }^{\circledR}$ lo que conllevó en una mejoría del rendimiento académico en esa parte del examen final.

Por otro lado, los datos relativos a las horas empleadas en el repaso de las dos partes del examen final revelan que la implantación de un método inductivo en el repaso de los ejercicios de cálculo de dosis aun no siendo estadísticamente significativo, indujo una reducción en las mismas. En este sentido, diversos autores han descrito que metodologías docentes como el aula-invertida induce una mayor retención de lo aprendido (Wong., 2014; McLean, 2016; White., 2017) lo cual podría explicar una menor necesidad de repasar los ejercicios en el curso académico 2018-2019. Unido a este hecho, la implantación de cuestionarios Socrative ${ }^{\circledR}$ ha conllevado que el alumnado haya repasado los materiales online para la parte teórica en mayor medida que en cursos anteriores, lo cual ha mejorado significativamente el rendimiento académico del alumnado.

Por tanto, podemos concluir: 1) la utilización de metodología inductiva y metodologías activas (cuestionarios Socrative ${ }^{\circledR}$ ), combinadas con la implantación de materiales on-line conlleva una mejora del rendimiento académico del alumnado en torno al cálculo de dosis. 2) el análisis del coste en horas empleadas por el alumnado en la realización de los ejercicios planteados revelan un resultado positivo ya que, aunque el aumento de horas empleadas no llegó a ser estadísticamente significativo, el rendimiento académico mejoró significativamente.

\section{Limitaciones del estudio}

La primera limitación de este estudio proviene que las horas empleadas en el repaso de los diferentes tipos de ejercicios es únicamente una aproximación a las horas reales empleadas por el alumnado, ya que no se han podido computar las horas previas de preparación y posteriores de estudio para el examen. Además, se ha constatado que algunos/as alumnos/as prefieren imprimir los materiales y realizarlos en papel. La segunda limitación viene derivada de la constatación de que el grupo de la línea idiomática de euskera en el curso académico 2018-2019 disponía de parte de las preguntas de la parte teórica del examen final 


\section{Agradecimientos}

El presente estudio ha sido financiado por el Servico de Asesoramiento Educativo (SAE/HELAZ) de la UPV/EHU a través del proyecto de innovación Educativo con código PIE-60.

\section{Referencias}

COUSINS DH, GERRETT y, WARNER B. (2011). "A review of medication incidents reported to the national reporting and learning system in England and Wales over 6 years" en British Journal and Clinical Pharmacology, vol. 74, issue 4, p. 597-604.

GARRO M A. RUBIO J.ZUBERO J. LACALLE J. y BARANDIARAN M T. (2018) "Mejora en la habilidad de cálculo de dosis a través de herramientas on-line" En: XV Foro Internacional sobre la Evaluación de la Calidad de la Investigación y de la Educación Superior (FECIES). 10-12 de Mayo Santander

GILL M, ANDERSEN E y HILSMANN N. (2019). "Best practices for teaching pharmacology to undergraduate nursing students: a systematic review of the literature" en Nurse Education Today, vol. 74 , p. $15-24$.

LACALLE J. RUBIO-PILARTE J. BARANDIARAN M T. AJA M N. y GARRO M A. (2019) "Recursos on-line en el aprendizaje de los efectos adversos en el grado de enfermería" En: VI Congreso Internacional de Docencia Universitaria. Vigo Comunicación aceptada

MANIAS E, KINNEY S, CRANSWICK N y WILLIAMS A. (2013). "Medication errors in hospitalized children" en Journal of Paediatrics and Child Health, vol. 50, p. 71-77.

McLEAN S, ATTARDI SM, FADEN L y GOLDSZMIDT M. (2016). "Flipped classrooms and student learning: not just surface gains" en Advances in Physiology Education, vol. 40, issue 1, p. 4755 .

WHITE PJ, NAIDU S, YURIEV E, SHORT JL, McLAUGHLIN JE y LARSON IC. (2017). "Student engagement with a flipped classroom teaching design affects pharmacology examination performance in a manner dependent on question type " en American Journal of Pharmacy Education, vol. 81, issue 9, p. 10-23.

WONG TH, IP EJ, LOPES I RAJAGOPALAN V y ABDELGUERFI M. (2014). "Pharmacy student's performance and perceptions in a flipped teaching pilot on cardiac arrhytmias" en American Journal of Pharmacy Education, vol. 78, issue 10, p. 1-6. 